\title{
BISAP SCORE: A SIMPLE TOOL TO ASSESS THE SEVERITY AND PREDICT THE MORBIDITY AND MORTALITY IN ACUTE PANCREATITIS
}

\author{
Hariprasad T. R', Haridarshan S. J2, Puneetha $K^{3}$, (Brig). S. Rajagopalan ${ }^{4}$ \\ ${ }^{1}$ Associate Professor, Department of General Surgery, Rajarajeshwari Medical College Hospital, Bangalore. \\ ${ }^{2}$ Assistant Professor, Department of General Surgery, Rajarajeshwari Medical College Hospital, Bangalore. \\ ${ }^{3}$ Post Graduate, Department of General Surgery, Rajarajeshwari Medical College Hospital, Bangalore. \\ 4 Professor and Head, Department of General Surgery, Rajarajeshwari Medical College Hospital, Bangalore.
}

ABSTRACT
NEED FOR STUDY
The present study is to use a simple bedside tool as a scoring system to assess the severity of acute pancreatitis and to predict its
risks for morbidity and mortality. The main criteria of this study is to highlight the ease of using this tool to identify the severity of
acute pancreatitis as early as possible in order to reduce the complications, risks and to improve the outcome and overall survival.

\section{MATERIALS AND METHODS}

Our study is a single centre, prospective observational study conducted at Rajarajeshwari Medical College Hospital, Bangalore, India from September 2014 to September 2015. Forty six patients, both males and females presenting within 24 hours of onset of symptoms diagnosed with acute pancreatitis were included in the study. Informed consents were obtained from all patients. Cases of chronic pancreatitis and acute on chronic pancreatitis were excluded. Pediatric patients of age less than 14 years and geriatric patients more than 70 years were excluded.

\section{RESULTS}

We observed that biliary pancreatitis was the most common with male population more affected than females. Pain abdomen was the most common presentation in the entire study population. Necrotizing pancreatitis was most commonly associated with ICU admission and prolonged hospital stay. Patients with organ failure and BISAP score more than 3 were found to have prolonged hospital stay. SIRS was the most common component of BISAP scoring system seen in $91.3 \%$. Elderly patients with age more than 60 years ( 6 pts.) had high BISAP score (5). All patients with shock had BISAP score of $>3$. A BISAP score of $\geq 3$ was associated with higher morbidity than scores of $<3$.

\section{CONCLUSION}

BISAP is a simple and a quick tool over other scoring systems and is similar to other scoring systems to predict the severity and prognosis of acute pancreatitis. In summary we have studied the ease of BISAP scoring system and its advantage in early recognition of acute pancreatitis, thus taking early steps to provide specific treatment to prevent complications, reduce the risks and to improve the overall survival.

\section{KEYWORDS}

Acute Pancreatitis; Scoring system; Bedside Index; Simple; Severity.

HOW TO CITE THIS ARTICLE: Hariprasad T. R, Haridarshan S. J, Puneetha K, (Brig). S. Rajagopalan. "Bisap Score: A Simple Tool to Assess the Severity and Predict the Morbidity and Mortality in Acute Pancreatitis." Journal of Evolution of Medical and Dental Sciences 2015; Vol. 4, Issue 101, December 17; Page: 16642-16645, DOI: 10.14260/jemds/2015/2484

\section{INTRODUCTION}

Acute pancreatitis is a process of acute inflammation of pancreas with peripancreatic tissue and multiorgan involvement and can have potentially devastating consequences. Most of the mild cases of acute pancreatitis resolve spontaneously without any sequelae, but in severe acute pancreatitis the mortality can go up to $30 \%{ }^{[1,2]}$ Hence, it is important to identify all cases of acute pancreatitis as early as possible to enable rapid intervention and treatment and thus improve patient's clinical condition and survival.

The understanding of the disease, its natural history and classification of pancreatic and peripancreatic fluid collections make the new 2012 Atlanta classification a valuable means to assess acute pancreatitis.

Financial or Other, Competing Interest: None.

Submission 26-11-2015, Peer Review 27-11-2015,

Acceptance 11-12-2015, Published 16-12-2015.

Corresponding Author:

Dr. Hariprasad T. $R$,

No. 507, $1^{\text {st }}$ C cross, $3^{\text {rd }}$ Main Road,

Banashankari $3^{\text {rd }}$ Stage, $2^{\text {nd }}$ Phase,

$6^{\text {th }}$ Block, Bangalore 560085.

E-mail: harishermi@hotmail.com

DOI:10.14260/jemds/2015/2484
This revised classification identifies 2 phases of acute pancreatitis - early (First 1 or 2 weeks) and late (Thereafter). Acute pancreatitis can be either oedematous interstitial pancreatitis or necrotizing pancreatitis, the latter involving necrosis of the pancreatic parenchyma and peripancreatic tissues, pancreatic parenchyma alone or just the peripancreatic tissues $(\sim 20 \%)$. Severity of the disease is categorized into 3 levels: mild, moderately severe and severe.

Mild acute pancreatitis lacks both organ failure (As classified by the modified Marshal scoring system) and local or systemic complications. Moderately severe acute pancreatitis has transient organ failure (Organ failure of $<2$ days), local complications and/or exacerbation of coexistent disease. Severe acute pancreatitis is defined by the presence of persistent organ failure (Organ failure that persists for $\geq 2$ days). Local complications are defined by objective criteria based primarily on contrast-enhanced computed tomography; these local complications are classified as acute peripancreatic fluid collections, pseudocyst (Which are very rare in acute pancreatitis), acute (Pancreatic/peripancreatic) necrotic collection and walled-off necrosis. ${ }^{[3]}$

Multiple scoring systems have been developed to assist early diagnosis and prognostication of acute pancreatitis. An 
ideal scoring system should promise an early, quick, simple, accurate and reproducible description of disease severity. Various scoring systems currently available are difficult to use and are poor predictors of the severity of acute pancreatitis. Ranson criteria, Acute Physiology And Chronic Health Evaluation (APACHE) II, Glasgow scoring system and Computed Tomography Severity Index (CTSI) are used in several institutions. Ranson's score requires 48 hours to complete the assessment and APACHE score is cumbersome to use.[4]

The main limitation of the Ranson criteria is that the evaluation cannot be completed until 48 hours following admission, which may lead to missing an early therapeutic window and increased mortality. ${ }^{[5]}$ Ranson's score is relatively accurate at classifying the severity of acute pancreatitis, but it is difficult to calculate the score as it requires a 48-hour missing the potential for early treatment.[5] The Acute Physiology And Chronic Health Examination (APACHE)-II was designed originally to predict intensive care unit survival and required the collection of many parameters, some of which might not be relevant to acute pancreatitis prognosis. ${ }^{[6]}$ CTSI is calculated based on CT findings of some local complications and cannot reflect the systemic inflammatory response.

In 2008, Wu et al. proposed a new simplified prognostic scoring system for the early determination of the severity of acute pancreatitis, namely 'bedside index of severity in acute pancreatitis' (BISAP). ${ }^{[7,8]}$ This system requires special attention since it is not universally accepted.

\section{AIM OF THE STUDY}

The aim is to study the feasibility of utilising BISAP score in identifying and assessing the severity of acute pancreatitis and in prediction the morbidity and mortality as early as possible, so that early intensive therapy can be instituted.

\section{MATERIALS AND METHODS}

Our study is a single centre, non-interventional, prospective observational study conducted at Raja Rajeshwari Medical College Hospital, Bangalore, India, for a period of one year from September 2014 to September 2015.

A total of 46 patients, both males and females presenting within 24 hours of onset of symptoms and diagnosed as acute pancreatitis supported by basic lab and imaging evidence were included in the study. Informed consents were obtained from each patient prior to enrolment. Cases of chronic pancreatitis and acute on chronic pancreatitis were excluded. Paediatric patients of age less than 14 years and geriatric patients more than 70 years were excluded.

\section{DIAGNOSTIC CRITERIA}

Acute pancreatitis was diagnosed based on the presence of characteristic abdominal pain; serum amylase level three times the upper limit of normal; the presence or absence of characteristic imaging findings of acute pancreatitis; and exclusion of other diseases. ${ }^{[9]}$

\section{RESULTS}

Forty six patients were enrolled in the study of which 32 were males and 14 were females [Fig. 1]. The youngest in the study was 21 years of age and the oldest was 70 years of age with a median age group of 45.5 years [Fig. 2]. The most common cause for acute pancreatitis in our study was biliary calculi in 12 males and 10 females followed by 16 males diagnosed with alcoholic pancreatitis followed by 2 males and 2 females each with post ERCP pancreatitis and finally 4 cases, 2 each of males and females with idiopathic pancreatitis [Fig. 3].

The most common presentation among these patients was pain abdomen followed by features of peritonitis, nausea and vomiting, abdominal distension and pleural effusion in the order of frequency [Fig. 4].

Mean duration of stay in the hospital was 9.5 days with the shortest duration being 3 days and the longest, 16 days [Fig. 5]. Eight patients progressed to multi-organ dysfunction syndrome with shock and 19 patients had multi-organ dysfunction without shock.

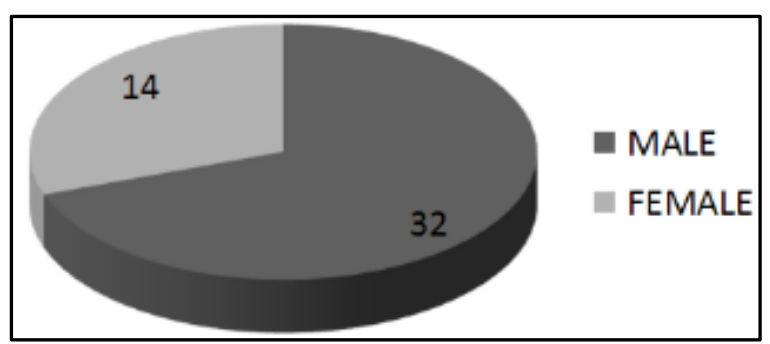

Fig. 1: [Sex Ratio]

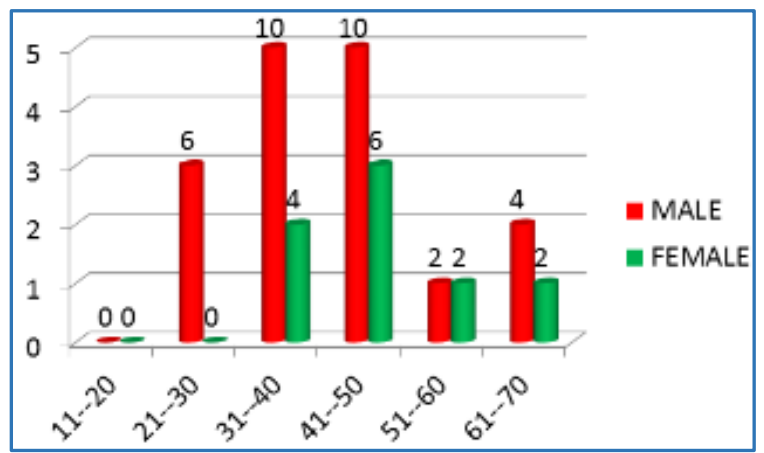

Fig. 2: [Age Distribution]

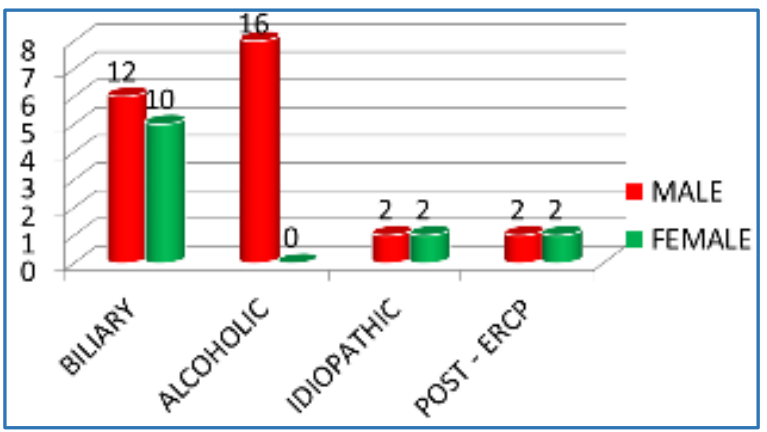

Fig. 3: [Aetiology]

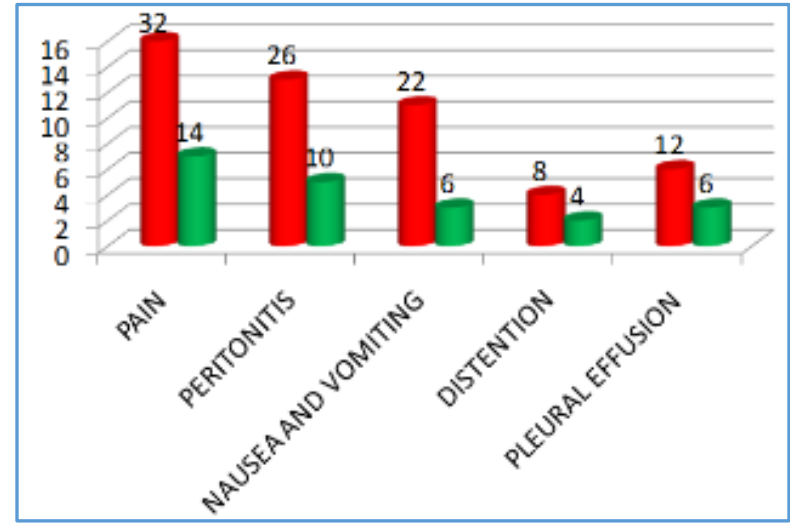

Fig. 4: [Clinical Presentation]

MALES FEMALES 


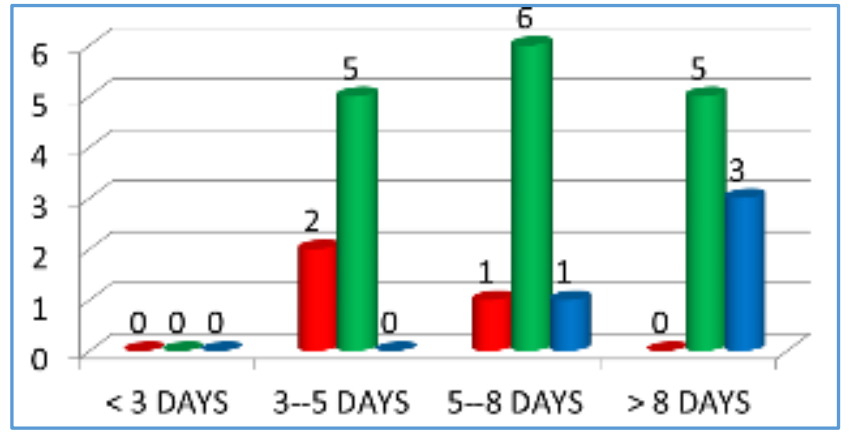

Fig. 5: [Duration of Stay based on severity]

BISAP <2 BISAP 2-4 BISAP 5

There were no cases of mortality in our study.
BISAP score among these patients were calculated and tabulated as follows [Tab. 1]

\begin{tabular}{|l|c|c|c|c|}
\hline CRITERIA & MALES & FEMALES & TOTAL & PERCENTAGE \\
\hline Blood Urea & & & & \\
Nitrogen & 6 & 4 & 10 & $21.7 \%$ \\
- $20-30$ & 16 & 6 & 22 & $47.8 \%$ \\
- $30-40$ & 10 & 4 & 14 & $30.4 \%$ \\
\hline $\begin{array}{l}\text { Impaired } \\
\text { Mental Status }\end{array}$ & 16 & 2 & 18 & $39.1 \%$ \\
Stupor/Coma & 4 & 0 & 4 & $8.6 \%$ \\
\hline SIRS & 30 & 12 & 42 & $91.3 \%$ \\
\hline Age >60 & 4 & 2 & 6 & $13 \%$ \\
\hline Pleural & 12 & 6 & 18 & $39.1 \%$ \\
Effusion & & & & \\
\hline
\end{tabular}

We compared our data with previous studies and the data was comparable [Tab. 2, Tab. 3]

\begin{tabular}{|c|c|c|c|c|}
\hline ETIOLOGY & Our Study (\%) & $\begin{array}{c}\text { Khanna et } \\
\text { al. (2013).[11] } \mathbf{( \% )}\end{array}$ & $\begin{array}{c}\text { Singh et al. } \\
\mathbf{( 2 0 0 9 ) . [ 8 ]}(\mathbf{\%})\end{array}$ & Chen et al. (2013).[10] (\%) \\
\hline Biliary & 47.8 & 61.1 & 27 & 66 \\
\hline Alcoholic & 34.8 & 18 & 21.4 & 6.8 \\
\hline Post ERCP & 8.7 & 2.8 & 14.6 & 1 \\
\hline Idiopathic & 8.7 & 12.5 & 15.1 & 16.1 \\
\hline
\end{tabular}

\begin{tabular}{|c|c|c|c|c|}
\hline BISAP SCORE & Our Study & Khanna et al. (2013) $^{[\mathbf{1 1}]}$ & Singh et al. (2009) $^{[\mathbf{8}]}$ & Chen et al. (2013) [10] (\%) $^{[\mathbf{\%}}$ \\
\hline$<3$ & $24(52 \%)$ & $36(50 \%)$ & $340(85.64 \%)$ & $396(79.6 \%)$ \\
\hline$>3$ & $22(48 \%)$ & $36(50 \%)$ & $57(14.35 \%)$ & $101(20.4 \%)$ \\
\hline TOTAL & $\mathbf{4 6}$ & $\mathbf{7 2}$ & $\mathbf{3 9 7}$ & $\mathbf{4 9 7}$ \\
\hline
\end{tabular}

We compared the severity of the disease, its complications, stay in the ICU and the duration of stay in our hospital with the study conducted by Khanna et al. (2013).[11]

\begin{tabular}{|c|c|c|}
\hline Parameters & Our Study[n=46] & $\begin{array}{c}\text { Khanna et al. } \\
{[\mathbf{n}=\mathbf{7 2}]^{[\mathbf{1 1}]}}\end{array}$ \\
\hline $\begin{array}{c}\text { SAP } \\
<3\end{array}$ & 4 & 8 \\
$>3$ & 12 & 23 \\
\hline OF & & \\
$<3$ & 8 & 5 \\
$>3$ & 28 & 20 \\
\hline Ne P & & 19 \\
$<3$ & 4 & 27 \\
$>3$ & 0 & 2 \\
\hline ICUA & 6 & 7 \\
$<3$ & & \\
$>3$ & 5 days & 7.5 days \\
\hline LOHS & 9 days & 10.2 days \\
\hline 3 &
\end{tabular}

SAP: Severe Acute Pancreatitis; OF: Organ Failure; Ne P: Necrotizing Pancreatitis; ICUA: ICU Admission; LOHS: Length of Hospital Stay.

\section{DISCUSSION}

A $10 \%$ to $20 \%$ of patients experienced a severe attack of acute pancreatitis resulting in an intense inflammatory response, a variety of local and systemic complications, a prolonged hospital course, and significant morbidity and mortality.[12] Modified Atlanta classification is a relatively objective index to evaluate both local and systemic changes in the development and progression of the disease.[13] There are multiple scoring systems available to assess the severity of acute pancreatitis.

Ranson and APACHE II scores are usually used to assess the severity of acute pancreatitis in most institutions.
A Ranson score of 3 or more or an APACHE II score of 8 or more indicates that the disease is quite severe with high chances of morbidity and mortality.

BISAP is a relatively new system used to predict the severity and prognosis of acute pancreatitis.[7] A study done by Papachristou et al.[14] reported a sensitivity of $37.5 \%$, specificity of $92.4 \%$, PPV of $57.7 \%$ and NPV of $84.3 \%$ towards prediction of severity of acute pancreatitis.

BISAP score has several advantages to assess the severity as compared to other scoring systems. The first is that the score is simple to calculate requiring only those vital signs, laboratory investigation studies and imaging that are commonly obtained at the time of presentation or within $24 \mathrm{~h}$ of presentation. The second is that the score was initially derived and tested using 36,248 cases of acute pancreatitis across 389 hospitals, reflecting the full spectrum of healthcare delivery. ${ }^{[7,8]}$ The third is that the score predicts in-hospital mortality.

BISAP comprises of 5 variables which can be obtained and calculated within 24 hours of admission namely,

- $\quad$ Blood Urea Nitrogen $(B U N)>25 \mathrm{mg} / \mathrm{dl}$.

- Impaired mental status (Disorientation, lethargy, somnolence, stupor or coma).

- $\quad \geq 2$ SIRS criteria. $[15,16]$

- $\mathrm{Temp}>38^{\circ} \mathrm{C}\left(100.4^{\circ} \mathrm{F}\right)$ or $<36^{\circ} \mathrm{C}\left(96.8^{\circ} \mathrm{F}\right)$.

- $\quad$ Heart Rate $>90$.

- $\quad$ Respiratory Rate $>20$ or PaCO2 $<32 \mathrm{mmHg}$.

- $\mathrm{WBC}>12,000 / \mathrm{mm}>3,<4,000 / \mathrm{mm}>3$ or $>10 \%$ bands.

- Age more than 60 years.

- $\quad$ Pleural effusion present. 


\begin{tabular}{|c|c|c|}
\hline PARAMETERS & Score 1 & Score 0 \\
\hline Blood Urea Nitrogen & $>25 \mathrm{mg} / \mathrm{dl}$ & $<25 \mathrm{mg} / \mathrm{dl}$ \\
\hline Impaired Mental Status & Present & Absent \\
\hline SIRS & $2 / 4$ present & Absent \\
\hline Age & $>60$ years & $<60$ years \\
\hline Pleural Effusion & Present & Absent \\
\hline
\end{tabular}

Any two of four will be significant if present simultaneously; A score of $>3$ will indicate severe pancreatitis (Early organ failure/pancreatic necrosis). [17] Both the BISAP and APACHE II scores incorporate systemic inflammatory response syndrome, age and Glasgow Coma Scale. However, with only the addition of blood urea nitrogen and pleural effusion the BISAP score has a discriminatory ability to predict mortality, which is equivalent to the APACHE II score.

A BISAP score of $\geq 3$ was associated with higher morbidity than scores of $<3$. BISAP scores of $\geq 3$ predicted the development of organ failure, persistent organ failure and pancreatic necrosis. ${ }^{[17]}$

As per Kim et al., BISAP is more accurate for predicting the severity of acute pancreatitis than the serum PCT, APACHE-II, Glasgow and CTSI scores. [18]

We observed in our study that biliary pancreatitis was the most common with male population more affected than females. Pain abdomen was the most common presentation in the entire study population. Necrotizing pancreatitis was most commonly associated with ICU admission and prolonged hospital stay. Patients with organ failure and BISAP score more than 3 were found to have prolonged hospital stay. SIRS was the most common component of BISAP scoring system seen in $91.3 \%$. Elderly patients with age more than 60 years ( 6 pts.) had high BISAP score (5). All patients with shock had BISAP score of $>3$.

\section{CONCLUSION}

Assessment of the severity of acute pancreatitis and identifying the risks for high morbidity and mortality early in the course of acute pancreatitis improves the overall outcome in patients to a large extent. BISAP score helps to assess the severity and risks of morbidity and mortality in acute pancreatitis as early as within 24 hours of presentation, which helps to improve the clinical care by early intervention, supportive measures and aggressive treatment. BISAP has the advantages of being simple to use and quick over traditional scoring systems, although performed similar to other scoring systems in predicting the severity of acute pancreatitis and the prognosis of acute pancreatitis. BISAP score is an accurate means for risk stratification and prognostic prediction in acute pancreatitis.

BISAP scoring as a pancreatic severity assessment tool is simpler and more predictive compared to Ranson's scoring.

BISAP scoring is more clinical and lesser laboratory based scoring system compared to other scoring systems.

In summary, we have studied the advantages of BISAP scoring system to assess the severity of acute pancreatitis and to predict the risks of morbidity and mortality, thus taking early steps to provide supportive measures and specific treatment to prevent complications, reduce the risks and to improve the overall survival.

\section{REFERENCES}

1. Banks PA, Freeman ML; Practice Parameters Committee of the American College of Gastroenterology. Am J Gastroenterol 2006;101:2379-2400.

2. Fagenholz PJ, Castillo CF, Harris NS, et al.; Increasing United States Hospital admissions for acute pancreatitis, 1988-2003.

Ann Epidemiol 2007;17:491-7.
3. Sarr MG; 2012 revision of the Atlanta classification of acute pancreatitis, Pol Arch Med Wewn 2013;123(3):118-24. Epub Jan 25.

4. Leung et al.; Balthazar computed tomography severity index is superior to Ranson criteria and APACHE II scoring system in predicting acute pancreatitis outcome, World Journal of Gastroenterology, 2005; Vol. II (38); 6049-6052.

5. Ranson JH, Pasternack BS. Statistical methods for quantifying the severity of clinical acute pancreatitis. J Surg Res 1977;22(2):79-91.

6. Knaus WA, Draper EA, Wagner DP, et al. APACHE II: a severity of disease classification system. Crit Care Med 1985;13:818-829.

7. Wu et al.; The early prediction of mortality in acute pancreatitis: a large population based study, Gut 2008;57:1698-1703.

8. Singh VK et al.; A prospective evaluation of the bedside index for severity in acute pancreatitis score in assessing mortality and intermediate markers of severity in acute pancreatitis. American Journal of Gastroenerology 2009;104:966-971.

9. The Pancreatology Working Group of Chinese Society of Gastroenterology of Chinese Medical Association. Draft criteria for diagnosis and treatment of acute pancreatitis in China. Mod Dig Interv 2007;12(3):206-208.

10. Chen, Lifen et al. "Evaluation of the BISAP Score in Predicting Severity and Prognoses of Acute Pancreatitis in Chinese Patients." International Surgery 98.1 (2013): 6-12.

11. Khanna, Ajay K et al. "Comparison of Ranson, Glasgow, MOSS, SIRS, BISAP, APACHE-II, CTSI scores, IL-6, CRP and procalcitonin in predicting severity, organ failure, pancreatic necrosis and mortality in acute pancreatitis." HPB Surgery 2013 (2013).

12. Servin-Torres E, Velazquez-Garcia JA, Delgadillo-Teyer G, Galindo-Mendoza L, Bevia-Perez F, Rivera-Bennet F. Severe acute pancreatitis: surgical management in a thirdlevel hospital. Cir Cir. 2009;77:407-410.

13. Stimac D, Miletić D, Radić M, Krznarić I, Mazur-Grbac M, Perković D, et al. The role of non-enhanced magnetic resonance imaging in the early assessment of acute pancreatitis. Am J Gastroenterol. 2007;102(5):997-1004.

14. Papachristou GI, Muddana V, Yadav D, O'Connell M, Sanders MK, Slivka A, et al. Comparison of BISAP, Ranson's, APACHE-II and CTSI scores in predicting organ failure, complications and mortality in acute pancreatitis. Am J Gastroenterol 2010;105(2):435-441.

15. Buter A, Imrie CW, Carter CR, et al. Dynamic nature of early organ dysfunction determines outcome in acute pancreatitis. Br J Surg 2002;89:298-302.

16. Mofidi R, Duff MD, Wigmore SJ, Madhavan KK, Garden OJ, Parks RW. Association between early systemic inflammatory response, severity of multiorgan dysfunction and death in acute pancreatitis. Br J Surg 2006;93:738-744.

17. Jayant L Pednekar, Sachin Patil. Bedside Index of Severity in Acute Pancreatitis (BISAP) score for predicting prognosis in acute pancreatitis 2015;2(2):62-70.

18. Kim BG, Noh MH, Ryu CH, Nam HS, Woo SM, Ryu SH, et al. A comparison of the BISAP score and serum procalcitonin for predicting the severity of acute pancreatitis. Korean J Intern Med 28(3):322-329. 